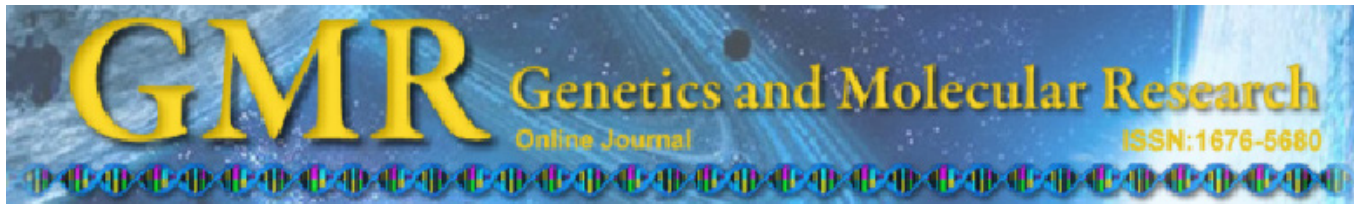

\title{
Association of NPP1 polymorphism with postoperative progression of ossification of the posterior longitudinal ligament in Chinese patients
}

\author{
Z. He ${ }^{1, *}$, H. Zhu ${ }^{1, *}$, L. Ding ${ }^{1}$, H. Xiao ${ }^{1}$, D. Chen ${ }^{2}$ and F. Xue ${ }^{1}$ \\ ${ }^{1}$ Department of Orthopaedics Surgery, \\ Shanghai Fengxian District Central Hospital, Shanghai, China \\ ${ }^{2}$ Department of Orthopaedics Surgery, Shanghai Changzheng Hospital, \\ Second Military Medical University, Shanghai, China \\ *These authors contributed equally to this study. \\ Corresponding authors: F. Xue / D. Chen \\ E-mail: fxdcspine@163.com
}

Genet. Mol. Res. 12 (4): 4648-4655 (2013)

Received November 23, 2012

Accepted July 5, 2013

Published October 18, 2013

DOI http://dx.doi.org/10.4238/2013.October.18.3

\begin{abstract}
Ossification of the posterior longitudinal ligament (OPLL) is a condition of the spine that can cause paralysis by compressing the spinal cord. The aim of this study was to evaluate the possible role of nucleotide pyrophosphatase phosphodiesterase 1 gene (NPP1) polymorphism in the etiology and pathology of the OPLL in Chinese patients. DNA from patients with OPLL $(\mathrm{N}=95)$ and without OPLL $(\mathrm{N}=90)$ were genotyped for 4 NPP1 single-nucleotide polymorphisms (SNPs): A533C, C973T, IVS15-14T $\rightarrow$ C, and IVS2011delT. An association study evaluated the relationship between specific SNP genotypes and susceptibility. We also evaluated whether genotypes of these SNPs were associated with disease severity and the probability of disease progression after surgery. The C973T and IVS1514T SNPs were associated with the existence of the disease. The TT genotypes of C973T and IVS15 $\rightarrow 14 \mathrm{~T}$ as well wild-type IVS20 (lack of deletion) were associated with more severe disease. Patients with the T
\end{abstract}


deletion of IVS20 or the AA genotype of A533C had an approximately 3 times greater chance of not having than having disease progression after surgery. We concluded that the 4 SNPs analyzed appeared to have different effects on the etiology and pathology of OPLL. To our knowledge, this study is the first to investigate the relationship between these SNPs and disease progression after surgery. Our findings suggest that the presence of specific genotypes of the IVS20-11delT and A533C SNPs may predict disease outcome after surgical intervention.

Key words: NPP1; Polymorphism; Ossification; Postoperative progression; Posterior longitudinal ligament

\section{INTRODUCTION}

Ossification of the posterior longitudinal ligament (OPLL) is an abnormal ossification of the ligament that runs behind the vertebral bodies in front of the spinal cord. It can result in compression of the spinal cord and lead to neurological symptoms that, in severe cases, require surgical treatment (Chang et al., 2012). OPLL occurs most commonly in men, in the elderly, and in Asians. The incidence of OPLL is $2.4 \%$ in Asians and $0.16 \%$ in non-Asians (Wang and Thambuswamy, 2011; Chang et al., 2012).

The exact pathogenesis and etiology of OPLL remains unclear. Early clinical and epidemiological studies have suggested that OPLL is multifactorial and results from both environmental and genetic factors (Terayama, 1989; Sakou et al., 1991). Several studies in Asian populations have indicated that collagen genes may be involved in OPLL (Koga et al., 1998; Maeda et al., 2001). Other factors include retinoic X receptor $\beta$, bone morphogenic protein, transforming growth factor $\beta$, insulin-like growth factors, connective tissue growth factors, growth hormone-binding proteins, platelet-derived growth factors, and interleukin-7 (Kon et al., 1997; Numasawa et al., 1999; Kawaguchi et al., 2003; Song et al., 2006).

The nucleotide pyrophosphatase phosphodiesterase multi-gene family (NPPS; also called ectophosphodiesterase/nucleotide phosphohydrolase) encodes nucleotide pyrophosphatases that are type II transmembrane metalloenzymes. NPP1, NPP2, and NPP3 regulate softtissue calcification and bone mineralization (Stapleton et al., 2011). The NPP1 protein isoform is the predominate enzyme in chondrocytes and osteoblasts that regulates bone mineralization (Goding et al., 2003). Four single-nucleotide polymorphisms (SNPs) in NPP1 appear to influence either the development or the severity of OPLL in humans. One is a $\mathrm{T} \rightarrow \mathrm{C}$ transition in intron 15 at position 14 upstream of exon 16 (IVS15-14T) (Koshizuka et al., 2002; Horikoshi et al., 2006) and another is a T deletion 11 nucleotides upstream of the splice acceptor site in intron 20 (IVS20-dell11T) (Tahara et al., 2005). The remaining 2 SNPs are variants in the coding region: $\mathrm{A} 533 \mathrm{C}$ is an $\mathrm{A}$ to $\mathrm{C}$ change in exon 4 that alters the protein sequence from $\mathrm{K}$ to $\mathrm{Q}$ (Nakamura et al., 1999), and C973T is a C to T substitution in exon 9.

Much of the genetic analysis of the association of NPP1 with OPLL has been performed in Japanese populations, and only a few prior studies investigated the association of NPP1 SNPs with the degree of disease severity (Koshizuka et al., 2002; Tahara et al., 2005). In this study, we evaluated the association of the 4 SNPs described above with susceptibility to OPLL, disease severity, and postoperative disease progression in a cohort of Chinese patients. 


\section{MATERIAL AND METHODS}

\section{Study population}

All patients in this study were treated at Shanghai Fengxian District Central Hospital and Shanghai Changzheng Hospital (China). The study was performed in accordance with the Declaration of Helsinki and was approved by the appropriate ethics committees. All patients gave their informed consent.

Patients included in this study had a confirmed diagnosis of OPLL and they were treated at the hospital before December 2006 (OPLL group). To establish a control group, we randomly selected individuals without OPLL from patients admitted to the hospital after January 2006. General conditions, imaging data, and follow-up information were recorded for all patients.

\section{Genotype analysis}

Blood samples $(5 \mathrm{~mL})$ were taken from patients in the OPLL and control groups and stored at $-20^{\circ} \mathrm{C}$. Subsequently, genomic DNA was extracted from the mononuclear cells of the blood samples using an EZ-10 Spin Column Bacterial Genomic DNA Isolation Kit (Sangon Biotech Co., Ltd., Shanghai, China) according to manufacturer instructions, and the purified DNA was stored at $-20^{\circ} \mathrm{C}$.

Genomic DNA was amplified using polymerase chain reaction (PCR) with a BS305 Ready-To-Use PCR Kit (Sangon Biotech) and sequenced to evaluate the presence of 4 SNPs: A533C, C973T, IVS15-14T $\rightarrow$ C, and IVS20-11delT. The PCR reaction included $2 \mu \mathrm{L}$ DNA template, $2 \mu \mathrm{L}$ each primer, $4 \mu \mathrm{L}$ deoxyribonucleotide triphosphate, $5 \mu \mathrm{L} 10 \mathrm{X}$ buffer, 0.3 $\mu \mathrm{L}$ Taq enzyme, and $34.7 \mu \mathrm{L}$ water. The PCR conditions for amplification of SNPs A533C, C973T, and IVS15-14T $\rightarrow \mathrm{C}$ were $94^{\circ} \mathrm{C}$ for 4 min followed by 35 cycles of $94^{\circ} \mathrm{C}$ for $40 \mathrm{~s}$, then $55^{\circ} \mathrm{C}$ for $30 \mathrm{~s}$ and $72^{\circ} \mathrm{C}$ for $40 \mathrm{~s}$ followed by 1 cycle of $72^{\circ} \mathrm{C}$ for $5 \mathrm{~min}$. The reaction conditions for IVS20-11delT were identical to that of the other SNPs, except that the annealing step was performed at $58^{\circ} \mathrm{C}$ instead of $55^{\circ} \mathrm{C}$. The primers used for PCR were presented in Table 1. The PCR products were sequenced by Shanghai Shengong Biotechnology Co. (China).

\begin{tabular}{llc}
\multicolumn{2}{l}{ Table 1. Information about the primers for the PCR. } \\
\hline SNPs & Sequence (5'-3') & Product size (bp) \\
\hline A533C & Forward: TGGGTGATGGGTATAATGT & 285 \\
C973T & Reverse: AACAGGCACAGAGGCTTCT & 314 \\
IVS15-14T $\rightarrow$ C & Forward: GGCCCACTTGTCTAGTTTT & 377 \\
IVS20-11delT & Reverse: GAGCAGAAAATGAAGGAAA & 374 \\
& Forward: TGAATCCACTTCCACAATA & 434 \\
\hline
\end{tabular}

\section{Imaging measurement and evaluation}

Lateral X-rays of the cervical spine was taken for all patients using a FUJI FCR AC-3 
CS Computed Radiography System, and the images were scanned and saved as electronic files with a UMAX Astra 1220S Scanner. OPLL length and thickness measurements in millimeters were obtained using SigmaScan Pro version 5 (SPSS Inc., USA). The details of the method are shown in Figure 1.

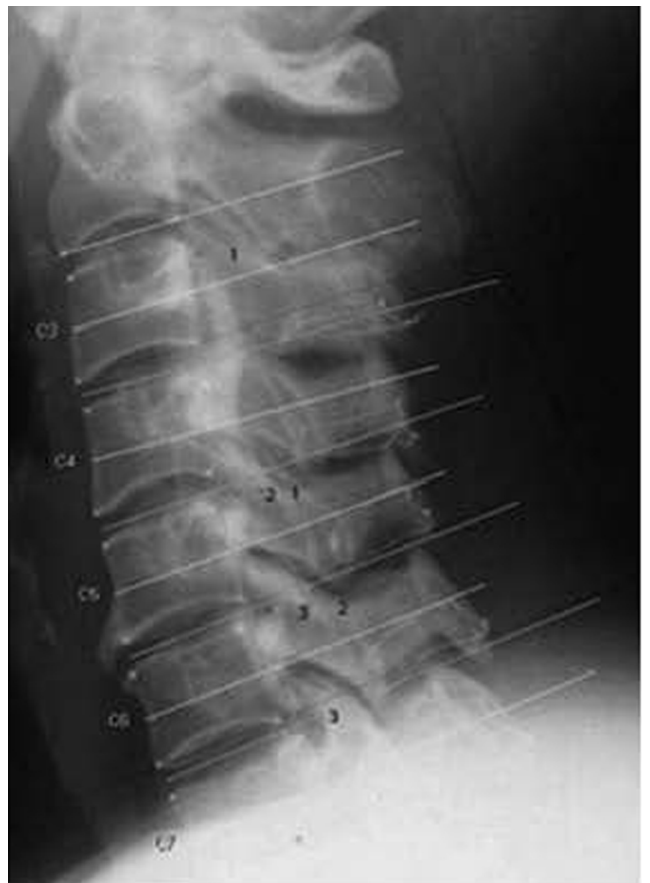

Figure 1. Radiographic measurement of ossification of the posterior longitudinal ligament. The patient was taken X-ray in lateral position at cervical spine position. The length and thickness of ossification of the posterior longitudinal ligament was measured. Millimeter is the unit for the measurement in the photo.

\section{Determination of ossification progression after cervical OPLL surgery}

OPLL length was measured according to the standard of Hori et al. $(4,5)$. The progression of OPLL thickness was defined as the extension of the upper or lower end of the ossified mass in the corresponding direction during follow-up visits. Progression was defined as greater than $2 \mathrm{~mm}$ of rearward growth. Otherwise, an assessment of no progression was recorded. Growth of a postoperative ossified mass (including the remnant after incomplete removal of the OPLL during decompression) more than $2 \mathrm{~mm}$ was considered progression. A newly developed ectopic ligament ossification greater than $2 \mathrm{~mm}$ in an area without previous ossification was considered progression. Growth of a remaining ossified mass more than $2 \mathrm{~mm}$ after partial resection of segmental ossification was also considered progression of ossification.

\section{Statistical analysis}

Data were presented as means \pm standard deviation for continuous variables and as number and percentage for categorical variables. For baseline characteristics or associations between SNPs 
and clinical characteristics, the comparisons of clinical characteristics between 2 groups were assessed with 2 independent sample $t$-tests for continuous variables. The chi-square test/Fisher exact test was used for categorical variables, and the magnitude of strength of association was reported as odds ratio. All statistical assessments were evaluated at a two-sided alpha level of 0.05. Analyses were performed with the SAS software package, version 9.2 (SAS Institute Inc., USA).

\section{RESULTS}

\section{Patient demographics and disease characteristics}

A total of 95 patients with diagnosed OPLL of the cervical spine and 90 control subjects were evaluated in this study. The OPLL and controls groups were mostly male ( $75.8 \%$ and $72.2 \%$, respectively) and the mean \pm SD ages were similar (56.3 \pm 9.1 and $57 \pm 8.7$, respectively).

The OPLL group consisted of patients with continuous OPLL $(\mathrm{N}=7)$, segmental OPLL $(\mathrm{N}=40)$, circumscribed OPLL $(\mathrm{N}=34)$, and mixed disease $(\mathrm{N}=14)$. Four patients in the OPLL group had combined anterior and posterior surgery. All patients were followed for 1-6 years and the mean length of follow-up was 3.1 years. All patients in the control group had only cervical spondylotic myelopathy without OPLL, diabetes, or ankylosing spondylitis.

\section{Genetic analysis}

Of the 4 SNPs analyzed, only the IVS20-11delT SNP was significantly out of HardyWeinberg equilibrium $(\mathrm{P}<0.001)$. The distribution of the genotypes of 2 of the SNPs, C973T and IVS15-14T $\rightarrow$ C, differed significantly between the OPLL and control groups (Table 2). Of subjects in the OPLL and control groups, $61.1 \%$ and $93.3 \%$, respectively, had the CC genotype of C973T, and $38.9 \%$ and $6.7 \%$, respectively, carried the TT+TC genotype $(\mathrm{P}<0.001)$. For IVS15-14T $\rightarrow \mathrm{C}$, the TT genotype occurred in the OPLL group more frequently than it did in the control group $(\mathrm{P}=0.026)$.

\begin{tabular}{lccc}
\multicolumn{2}{l}{ Table 2. Association between SNP genotypes and OPLL. } & \\
\hline Genotype [N (\%)] & $\begin{array}{c}\text { OPLL } \\
(\mathrm{N}=95)\end{array}$ & $\begin{array}{c}\text { Control } \\
(\mathrm{N}=90)\end{array}$ & $\mathrm{P}$ \\
\hline A533C & & - & 0.430 \\
AA & $76(80.0)$ & $76(84.4)$ & - \\
AC+CC & $19(20.0)$ & $14(15.6)$ & - \\
C973T & $58(61.1)$ & $84(93.3)$ & - \\
CC & $37(38.9)$ & $6(6.7)$ & - \\
TT+TC & $18(19.0)$ & - & 0.026 \\
IVS15-14T $\rightarrow$ C & $77(81.0)$ & $83(7.8)$ & - \\
TT & $55(57.9)$ & - & - \\
CC+TC & $40(42.1)$ & $41(45.6)$ & - \\
IVS20-11delT & & & - \\
WW & & $49(54.4)$. & - \\
VV & & & - \\
\hline V
\end{tabular}

$\mathrm{V}=$ variant; $\mathrm{W}=$ wild type.

Three of the 4 SNPs were associated with characteristics of OPLL disease severity that included ossified segments and thickness (Table 3). For SNP C973T, the homozygous $\mathrm{CC}$ genotype was associated with smaller ossification thickness compared with that of the 
$\mathrm{TT}+\mathrm{TC}$ genotypes $(\mathrm{P}=0.007)$. For IVS15-14T $\rightarrow \mathrm{C}$, patients with the TT genotype had greater numbers of ossified vertebrae and thicker ossification than those of patients with the CC+TC genotypes (P values $\leq 0.017$ ). Patients homozygous for the T deletion $(V V)$ in IVS20 had fewer ossified vertebrae and less ossification thickness than those in patients homozygous for the wild-type allele (WW; all P values $<0.001$ ).

\begin{tabular}{|c|c|c|c|c|}
\hline SNPs & $\mathrm{N}$ & $\begin{array}{c}\text { Age } \\
{[\text { means } \pm \mathrm{SD},(\text { years })]}\end{array}$ & $\begin{array}{c}\text { Ossified segments } \\
{[\text { means } \pm \text { SD, (VB)] }}\end{array}$ & $\begin{array}{c}\text { Ossified thickness } \\
{[\text { means } \pm \mathrm{SD},(\mathrm{mm})]}\end{array}$ \\
\hline \multicolumn{5}{|l|}{ A533C } \\
\hline AA & 76 & $55.7 \pm 9.0$ & $4.2 \pm 1.7$ & $7.8 \pm 3.1$ \\
\hline $\mathrm{AC}+\mathrm{CC}$ & 19 & $58.5 \pm 9.4$ & $4.7 \pm 2.5$ & $7.7 \pm 3.3$ \\
\hline P value & & 0.231 & 0.363 & 0.947 \\
\hline \multicolumn{5}{|l|}{ C973T } \\
\hline $\mathrm{CC}$ & 58 & $56.1 \pm 9.0$ & $4.1 \pm 1.8$ & $7.1 \pm 2.8$ \\
\hline $\mathrm{TT}+\mathrm{TC}$ & 37 & $56.5 \pm 9.3$ & $4.6 \pm 2.1$ & $8.8 \pm 3.3$ \\
\hline$P$ value & & 0.828 & 0.248 & 0.007 \\
\hline \multicolumn{5}{|c|}{ IVS15-14T $\rightarrow$ C } \\
\hline TT & 18 & $58.7 \pm 11$ & $6.3 \pm 2.4$ & $9.3 \pm 3.4$ \\
\hline $\mathrm{CC}+\mathrm{TC}$ & 77 & $55.7 \pm 8.6$ & $3.8 \pm 1.4$ & $7.4 \pm 2.9$ \\
\hline$P$ value & & 0.219 & $<0.001$ & 0.017 \\
\hline \multicolumn{5}{|c|}{ IVS20-11 delT } \\
\hline WW & 55 & $57.5 \pm 9.4$ & $5.1 \pm 4.5$ & $8.8 \pm 3.0$ \\
\hline VV & 40 & $54.6 \pm 8.5$ & $3.9 \pm 2.9$ & $6.4 \pm 2.7$ \\
\hline$P$ value & & 0.119 & $<0.001$ & $<0.001$ \\
\hline
\end{tabular}

Results are reported as means $\pm \mathrm{SD}$. Comparisons between two groups were performed by two sample independent $t$-test. $\mathrm{V}=$ variant; $\mathrm{W}=$ wild type.

We analyzed the association of disease progression after surgery with the 4 SNPs. The AA genotypes of SNP A533C and variant IVS20 (VV; T deletion) were associated with a greater proportion of patients without disease progression compared with that in patients with disease progression (both $\mathrm{P}$ values $<0.030$; Table 4). For both of these genotypes, patients were 3 times more likely to display no disease progression after surgery. The AC genotype of A533C and the WW genotype of IVS20-11delT seemed to have no influence on disease progression, as a similar proportion of patients with these genotypes were found in the progression and no-progression groups after surgery. For C973T and IVS15-14T $\rightarrow$ C, none of the analyzed genotypes were significantly associated with disease progression.

\begin{tabular}{|c|c|c|c|c|}
\hline & Non-progression $(\mathrm{N}=56)$ & Progression $(\mathrm{N}=39)$ & $P$ value & OR $(95 \% \mathrm{CI})$ \\
\hline A533C & & & 0.029 & \\
\hline AA & $49(87.5)$ & $27(69.2)$ & & $3.11(1.10,8.84)$ \\
\hline $\mathrm{AC}+\mathrm{CC}$ & $7(12.5)$ & $12(30.8)$ & & 1 \\
\hline C973T & & & 0.935 & \\
\hline $\mathrm{CC}$ & $34(60.7)$ & $24(61.5)$ & & $0.97(0.42,2.23)$ \\
\hline $\mathrm{TT}+\mathrm{TC}$ & $22(39.3)$ & $15(38.5)$ & & 1 \\
\hline IVS15-14T $\rightarrow C$ & & & 0.836 & \\
\hline TT & $11(19.6)$ & $7(18.0)$ & & $0.90(0.31,2.56)$ \\
\hline $\mathrm{CC}+\mathrm{TC}$ & $45(80.4)$ & $32(82.0)$ & & 1 \\
\hline IVS20-11delT & & & 0.007 & \\
\hline WW & $26(46.4)$ & $29(74.4)$ & & 1 \\
\hline VV & $30(53.6)$ & $10(25.6)$ & & $3.35(1.37,8.15)$ \\
\hline
\end{tabular}

Results presented as count and percentage. Comparisons between two groups were performed by chi-square test. Odds ratio $(\mathrm{OR})$ was used to expression the correlation. $\mathrm{V}=$ variant; $\mathrm{W}=$ wild-type. 


\section{DISCUSSION}

In this study, we found that several SNPs were associated with distinct OPLL clinical phenotypes, suggesting they may play roles in the etiology and pathology of OPLL. The C973T SNP was associated with disease susceptibility and severity but not with disease progression after surgery, as the distribution of the genotypes differed between OPLL and control patients, and the TT genotype was associated with thicker ossification. The IVS15-14T $\rightarrow$ C SNP was also associated with disease susceptibility and progression. The distribution of IVS15-14T $\rightarrow$ C genotypes was significantly different between the 2 patient groups, and patients homozygous for the $\mathrm{CC}$ allele had fewer ossified vertebrae and thinner ossification than those in patients carrying the TT genotype. In contrast, A533C and IVS20-del11T were not associated with disease susceptibility. However, the IVS20 locus did appear important both for disease severity and progression; patients without the deletion had more ossified vertebrae and thicker ossification than those in patients carrying the deletion, and they had a 3 times greater chance of displaying no disease progression after surgery. This SNP was also out of HardyWeinberg equilibrium, suggesting that some selection pressures may be at work on this locus. The A533C SNP may also play a role in disease progression, because patients homozygous for the A allele also had an approximately 3 times greater probability of disease progression.

The relationships of IVS15-14T and IVS20-11delT with disease severity have previously been studied (Koshizuka et al., 2002; Tahara et al., 2005). These studies have found that the association with severity was influenced by gender (Koshizuka et al., 2002; Tahara et al., 2005 ). Women with early onset disease and the $\mathrm{C}$ allele of IVS15-14T $\rightarrow \mathrm{C}$ had a greater number of ossified vertebrae than that in non-carriers $(\mathrm{P}<0.05)$ (Koshizuka et al., 2002). In men, this relationship of the $C$ allele and disease severity was unapparent (Koshizuka et al., 2002). For IVS20-11delT, men but not women had a greater number of ossified vertebrae than that in patients without the deleted T allele $(\mathrm{P}<0.05)$ (Tahara et al., 2005). We did not evaluate the role of gender in the phenotype of the studied alleles, although most of our population was male.

Consistent with our findings, 2 studies have found that the IVS20-11delT polymorphism is unassociated with the disease (Koshizuka et al., 2002; Tahara et al., 2005). However, in another study, the proportion of patients with the T allele deletion in the OPLL group was significantly higher than that in controls (0.0029), suggesting this allele is unassociated with susceptibility (Nakamura et al., 1999).

The reason for the differences between our findings and those other studies is unclear, but it may represent variations among populations (e.g., ethnicity, ratio of men to women), the small sample size of all the studies, and the fact that other studies have evaluated the relationship of specific SNP alleles and disease parameters, whereas our study assessed genotypes.

The sample size of this study was small, and larger studies are necessary to confirm our findings. In addition, our study population was predominately male (about $75 \%$ ), and because some of SNP effects are gender specific, a study that evaluates a greater proportion of women is warranted.

In summary, our findings are consistent with others suggesting that $N P P 1$ is important to the etiology and pathology of OPLL. How the 4 SNPs investigated in this study alter bone metabolism to affect bone formation is unknown. To our knowledge, this study is the first to investigate the relationship of these SNPs to disease progression after surgery. Our findings suggest that the presence of specific genotypes of the IVS20-11delT and A533C SNPs may 
predict the prognosis of patients after surgical intervention.

\section{Conflicts of interest}

The authors declare no financial conflict with any person or organization.

\section{REFERENCES}

Chang H, Song KJ, Kim HY and Choi BW (2012). Factors related to the development of myelopathy in patients with cervical ossification of the posterior longitudinal ligament. J. Bone Joint Surg. Br. 94: 946-949.

Goding JW, Grobben B and Slegers H (2003). Physiological and pathophysiological functions of the ecto-nucleotide pyrophosphatase/phosphodiesterase family. Biochim. Biophys. Acta 1638: 1-19.

Horikoshi T, Maeda K, Kawaguchi Y, Chiba K, et al. (2006). A large-scale genetic association study of ossification of the posterior longitudinal ligament of the spine. Hum. Genet. 119: 611-616.

Kawaguchi Y, Furushima K, Sugimori K, Inoue I, et al. (2003). Association between polymorphism of the transforming growth factor-betal gene with the radiologic characteristic and ossification of the posterior longitudinal ligament. Spine 28: 1424-1426.

Koga H, Sakou T, Taketomi E, Hayashi K, et al. (1998). Genetic mapping of ossification of the posterior longitudinal ligament of the spine. Am. J. Hum. Genet. 62: 1460-1467.

Kon T, Yamazaki M, Tagawa M, Goto S, et al. (1997). Bone morphogenetic protein-2 stimulates differentiation of cultured spinal ligament cells from patients with ossification of the posterior longitudinal ligament. Calcif. Tissue Int. 60: 291-296.

Koshizuka Y, Kawaguchi H, Ogata N, Ikeda T, et al. (2002). Nucleotide pyrophosphatase gene polymorphism associated with ossification of the posterior longitudinal ligament of the spine. J. Bone Miner. Res. 17: 138-144.

Maeda S, Koga H, Matsunaga S, Numasawa T, et al. (2001). Gender-specific haplotype association of collagen alpha2 (XI) gene in ossification of the posterior longitudinal ligament of the spine. J. Hum. Genet. 46: 1-4.

Nakamura I, Ikegawa S, Okawa A, Okuda S, et al. (1999). Association of the human NPPS gene with ossification of the posterior longitudinal ligament of the spine (OPLL). Hum. Genet. 104: 492-497.

Numasawa T, Koga H, Ueyama K, Maeda S, et al. (1999). Human retinoic X receptor beta: complete genomic sequence and mutation search for ossification of posterior longitudinal ligament of the spine. J. Bone Miner. Res. 14: 500-508.

Sakou T, Taketomi E, Matsunaga S, Yamaguchi M, et al. (1991). Genetic study of ossification of the posterior longitudinal ligament in the cervical spine with human leukocyte antigen haplotype. Spine 16: 1249-1252.

Song J, Mizuno J, Hashizume Y and Nakagawa H (2006). Immunohistochemistry of symptomatic hypertrophy of the posterior longitudinal ligament with special reference to ligamentous ossification. Spinal Cord 44: 576-581.

Stapleton CJ, Pham MH, Attenello FJ and Hsieh PC (2011). Ossification of the posterior longitudinal ligament: genetics and pathophysiology. Neurosurg. Focus 30: E6.

Tahara M, Aiba A, Yamazaki M, Ikeda Y, et al. (2005). The extent of ossification of posterior longitudinal ligament of the spine associated with nucleotide pyrophosphatase gene and leptin receptor gene polymorphisms. Spine 30: 877-880.

Terayama K (1989). Genetic studies on ossification of the posterior longitudinal ligament of the spine. Spine 14: 11841191.

Wang MY and Thambuswamy M (2011). Ossification of the posterior longitudinal ligament in non-Asians: demographic, clinical, and radiographic findings in 43 patients. Neurosurg. Focus 30: E4. 